\title{
The joint flanker effect: sharing tasks with real and imagined co-actors
}

\author{
Silke Atmaca • Natalie Sebanz $\cdot$ Günther Knoblich
}

Received: 15 December 2010/ Accepted: 20 April 2011/Published online: 15 May 2011

(C) The Author(s) 2011. This article is published with open access at Springerlink.com

\begin{abstract}
The Eriksen flanker task (Eriksen and Eriksen in Percept Psychophys 16:143-149, 1974) was distributed among pairs of participants to investigate whether individuals take into account a co-actor's S-R mapping even when coordination is not required. Participants responded to target letters (Experiment 1) or colors (Experiment 2) surrounded by distractors. When performing their part of the task next to another person performing the complementary part of the task, participants responded more slowly to stimuli containing flankers that were potential targets for their co-actor (incompatible trials), compared to stimuli containing identical, compatible, or neutral flankers. This joint Flanker effect also occurred when participants merely believed to be performing the task with a coactor (Experiment 3). Furthermore, Experiment 4 demonstrated that people form shared task representations only when they perceive their co-actor as intentionally controlling her actions. These findings substantiate and generalize earlier results on shared task representations and advance our understanding of the basic mechanisms subserving joint action.
\end{abstract}

Keywords Joint action - Task sharing - Shared representations $\cdot$ Action anticipation

\footnotetext{
S. Atmaca

Department of Psychology, Max Planck Institute for Human Cognitive and Brain Sciences, Leipzig, Germany

N. Sebanz · G. Knoblich $(\bowtie)$

Centre for Cognition, Donders Institute for Brain, Cognition, and Behaviour, Radboud University Nijmegen, P.O. Box 9104, 6500 HE Nijmegen, The Netherlands

e-mail: G.Knoblich@donders.ru.nl
}

\section{Introduction}

In many situations, people act in close proximity to one another be it in a factory, in an open-plan office, cycling in the Tour de France, or simply walking along the street. Even when people act alone and do not intend to coordinate with others, their performance is shaped by others' actions. This general principle manifests itself in a variety of different phenomena, ranging from social facilitation over entrainment and perception-action interference to task co-representation.

The term 'social facilitation' refers to the phenomenon that people perform (simple) tasks more quickly in the mere presence of others (Aiello and Douthitt 2001; Guerin 1993; Zajonc 1965). This finding holds in situations where neither cooperation nor competition is critical for task performance and is thought to be due to differences in arousal.

More recently, research on interpersonal entrainment has revealed that when people perform cyclic actions like swinging pendulums (Amazeen et al. 1995; Richardson et al. 2005; Schmidt et al. 1998), walking (van Ulzen et al. 2008), or rocking in rocking chairs (Richardson et al. 2007) next to each other, they tend to fall into the same rhythm. This indicates that dynamical principles of intrapersonal coordination also hold for the interpersonal case (Marsh et al. 2009).

A third way in which others' actions affect performance is demonstrated by studies of perception-action interference. People are faster and more accurate at performing actions when they concurrently observe someone else performing the same action, and they are slower and less accurate when they see another person performing an opposite action (Brass et al. 2000, 2001; Kilner et al. 2003; Longo et al. 2008; Stuermer et al. 2000). For example, 
participants can lift their index finger more quickly when seeing someone lifting their index finger compared to seeing the middle finger being lifted (Brass et al. 2001). These results indicate that observing an action activates motor programs also involved in performing the same action, as proposed by common coding theory (Prinz 1997). The notion of common representations for action performance and action perception is well supported by neurophysiological and brain-imaging studies (for an overview, see Rizzolatti and Sinigaglia 2010).

Finally, cognitive and social scientists have started to distribute tasks between two co-actors to investigate whether individuals form similar task representations when task performance is shared and when the whole task is performed alone. The terms 'task co-representation' and 'shared task representations' refer to the idea that during joint task performance, each co-actor represents not only her own part, but also the part to be performed by the co-actor.

In a first study, Sebanz et al. (2003) distributed a spatial compatibility task between two people to test whether individuals integrate a co-actor's action alternative in their action planning. In the standard version of this task (e.g., Simon 1990), a single person performs right and left key presses in response to targets of two different colors (twochoice RT task). For instance, red targets might require a left response, and green targets a right response. The targets occur in different positions on the screen (e.g., left side vs. right side), yet target position is irrelevant to task performance. Nonetheless, the irrelevant spatial dimension of the stimuli interferes with responses. Reaction times (RTs) are faster when a stimulus appears on the side of the response to be given (compatible trials, e.g., red target appearing on the left) compared to when the stimulus appears on the opposite side of the response (incompatible trials). This compatibility effect is explained by the dimensional overlap model (Kornblum et al. 1990; Kornblum and Lee 1995), according to which the irrelevant spatial feature of the stimulus triggers an automatic response activation because the irrelevant stimulus dimension and features of the response overlap. On incompatible trials, the automatic response activation leads to response interference, resulting in longer RTs.

In the new, social version, one person was in charge of the left response key and the other was in charge of the right response key (similar as in Fig. 1, bottom). The crucial question was whether interference between the left and the right response that normally occurs within participants would also be found across participants. The results of this initial study and several further studies (e.g., Milanese et al. 2010; Welsh 2009) confirm this prediction. When participants responded to stimuli of a particular color, they were faster in doing so when the stimuli appeared in a spatial location that corresponded to the side of the response location, compared to when the stimuli appeared on the opposite side. This pattern was found both when participants acted alone (two-choice condition), choosing between a left and a right response, and when they acted
Fig. 1 Illustration of neutral trials (left column) and incompatible trials (right column) in the individual condition (top row) and joint condition (bottom row). In the joint condition, the flankers on incompatible trials were part of the co-actor's task rules

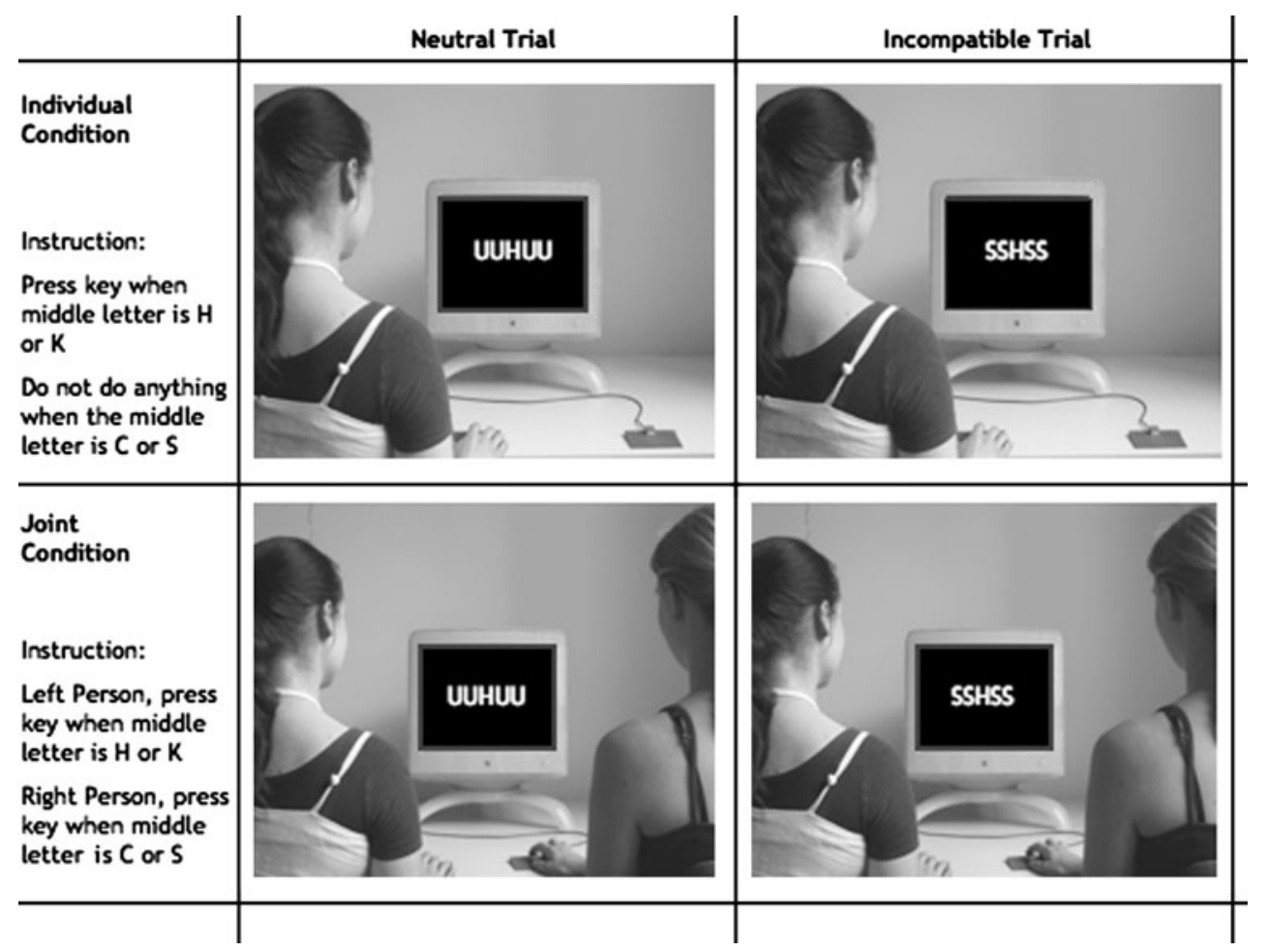


together (joint go/no-go condition), with each individual in the pair taking care of only one of the two possible responses. Importantly, this spatial compatibility effect was not observed when participants performed their half of the task alone without the co-actor (individual go/no-go condition).

Several studies suggest that task co-representation effects occur even when the co-actor is invisible (Ruys and Aarts 2010; Sebanz et al. 2005; Tsai et al. 2008; Vlainic et al. 2010, but see Welsh et al. 2007 for a contradictory result). This indicates that knowledge about a co-actor's task may be sufficient to modulate individual action planning. However, it is unclear whether task co-representation effects are restricted to tasks involving automatic stimulusresponse (S-R) links, such as the Simon task, or whether they are more general. Is a representation of an action under the control of one's co-actor only activated through direct S-R links, or do co-actors represent each other's actions in such a way that even arbitrary $\mathrm{S}-\mathrm{R}$ links can lead to activation of the co-actor's response?

Findings by Atmaca et al. (2008) indicate that co-representation effects can be generalized to a task where spatial S-R links are more indirect. In a joint SNARC paradigm, one participant responded to odd numbers and the other to even numbers, presented centrally on a computer screen. It is known that individuals performing the whole task alone are faster responding to small numbers with a left key press and to large numbers with a right key press, indicating that numbers are represented in terms of magnitude on a mental number line proceeding from left to right (Dehaene et al. 1993). The same SNARC effect was found when participants performed the task together, suggesting that co-representation effects can be generalized to tasks where the spatial overlap between stimuli and responses is indirect.

Sebanz et al. (2005) found that a nonspatial task interfered with a spatial task; participants responding to a spatial stimulus feature showed larger reaction times when their co-actor responded to stimulus color at the same time. However, this study focused on actions performed simultaneously. It is unknown whether performance of actions carried out while the other actor is idle is also affected by knowledge about the other's task rules. Furthermore, task rules assignment was not completely arbitrary, making it difficult to generalize the results.

The aim of the present study was to investigate whether co-representation effects occur in the absence of automatic $\mathrm{S}-\mathrm{R}$ links. Both in the joint Simon and in the joint SNARC task, irrelevant stimulus features (spatial position and numerosity, respectively) directly trigger activations of the co-actor's response. In the present study, we used the Eriksen flanker task (Eriksen and Eriksen 1974; see explanations below), where links between stimuli and responses are arbitrary (instructed). According to the dimensional overlap model (Kornblum and Lee 1995), the feature overlap in the Simon and in the SNARC task occurs between the response set and the irrelevant stimulus dimension (type 3 in their taxonomy). In contrast, the overlap in the Flanker task occurs between the relevant and an irrelevant stimulus dimension (type 4). The occurrence of a joint Flanker effect would thus provide evidence that co-actors represent the (arbitrary) task rules that govern their partner's performance.

Experiments 1 and 2 investigated the joint Flanker effect with two different sets of stimuli. In two further experiments, we asked which contextual factors determine whether co-actors represent each other's tasks. Experiment 3 investigated the role of the co-actor's presence, and Experiment 4 tested whether perceiving the co-actor as acting intentionally is a necessary factor. Before reporting the experiments, we shortly introduce the Flanker paradigm.

\section{The Flanker task}

The Flanker task (Eriksen and Eriksen 1974) is a wellestablished experimental paradigm in cognitive psychology. For more than three decades, it has been used in attempts to separate different processing levels in hierarchical stage models of cognitive processing (Eriksen and Schultz 1979; Miller 1988; for a critical discussion, see Cohen and Shoup 1997) and in the study of response selection processes in particular (Coles et al. 1985; Grice and Gwynne 1985; Sanders and Lamers 2002). More recently, the task has been extensively used in studies of conflict resolution and error monitoring (e.g., Botvinick et al. 2001; Van Veen et al. 2001).

In the standard Flanker task, participants respond to different targets (e.g., letters, shapes, colors, etc.) by pressing one of two different keys. Targets are surrounded by distracting flankers that are either (1) the same as the target (identical trials), (2) perceptually different from the target but refer to the same response (compatible trials), (3) perceptually different and refer to the opposite response as the target (incompatible trials), or (4) perceptually different and do not refer to any response (neutral trials). Note that the flankers in (1), (2), and (3) may appear as targets on other trials, whereas the flankers in (4) never appear as targets (see Table 1 for the full set of target-flanker combinations used in Experiments 1, 3, and 4).

Participants' RTs vary depending on the combination of target and flankers. RTs on neutral trials provide a reference value, because flankers do not refer to any response. For instance, if the task is to respond to the letters $\mathrm{S}$ and $\mathrm{C}$ with a left response and the letters $\mathrm{H}$ and $\mathrm{K}$ with a right response, the flanker $U$ in the stimulus UUKUU will 
Table 1 Complete stimulus set of Experiments 1, 3, and 4

\begin{tabular}{cllll}
\hline & Identical & Compatible & Neutral & Incompatible \\
\hline Target & & & & \\
H & HHHHH & KKHKK & UUHUU & SSHSS \\
K & KKKKK & HHKHH & UUKUU & CCKCC \\
S & SSSSS & CCSCC & UUSUU & KKSKK \\
C & CCCCC & SSCSS & UUCUU & HHCHH \\
\hline
\end{tabular}

The letters " $H$ " and " $K$ " were assigned to one response key, the letters " $\mathrm{S}$ " and " $\mathrm{C}$ " were assigned to the other response key. Letters were presented white on black

activate neither the right response nor the left response. Compared to neutral trials, responses on compatible trials are usually faster because flankers provide additional activation of the response required by the target and thus facilitate response selection (e.g., Eriksen and Schultz 1979). For instance, in the stimulus HHKHH, the flanker $H$ also activates the right response required by the target $\mathrm{K}$. RTs on identical trials are usually even faster than RTs on compatible trials. In addition to activating the same response as the target, perceptual processing is typically facilitated because identical flankers provide additional perceptual information (Cohen and Shoup 1997; Eriksen and Eriksen 1979), such as in the stimulus KKKKK. Finally, compared to neutral trials, RTs on incompatible trials are slower because flankers activate the opposite response as the target, which creates response interference (Eriksen and Eriksen 1979; Eriksen and Schultz 1979). For instance, in the stimulus SSKSS, the flanker S activates the left response, whereas the target $\mathrm{K}$ requires the right response. Note that, unlike in the Simon task where the irrelevant spatial stimulus features directly activates a spatially corresponding response, flankers can only activate a response if the instructed S-R mappings are known to a participant. The Flanker task is thus optimally suited for investigating whether co-actors take into account each other's task rules.

\section{The joint flanker task-predictions}

We distributed the Flanker task among pairs of participants so that each participant responded to two targets (letters in Experiments 1, 3, 4; colors in Experiment 2) with only one response, respectively. Participants performed the same go/ no-go task on their own (individual condition) and next to a co-actor (joint condition). We predicted a larger difference between RTs on incompatible trials and all other trials types (identical, compatible, neutral) in the joint condition compared to the individual condition. The reasoning for this prediction is as follows: In the joint condition, incompatible flankers require the co-actor's response when they occur in the target position. Due to the overlap between relevant (target) and irrelevant (flankers) stimulus features, incompatible flankers should trigger a representation of the other's action, interfering with response selection.

The dimensional overlap model predicts that in the individual go/no-go condition, a residual Flanker effect should also be present. While in the individual Simon go/ no-go task response interference does not occur when only one response is available, in the individual Flanker go/nogo task, there is still conflict between relevant and irrelevant stimulus features. This conflict should be more pronounced on incompatible trials compared to neutral trials. Neutral flankers never appear in the target position, whereas incompatible flankers do, so that neutral flankers will not activate as strong a tendency to inhibit a response as incompatible flankers. To illustrate this, imagine a participant responding to " $\mathrm{H}$ " and " $\mathrm{K}$ " in the individual condition. In the no-go trial "CCSCC", the letter "S" requires inhibition. When the same letter is flanking the target, as in the incompatible stimulus "SSHSS", "S" is still associated with action inhibition, even though " $\mathrm{S}$ " is not explicitly assigned to a competing action. Hence, even in the individual condition, RTs on incompatible trials should be slower than RTs on neutral trials (Heil et al. 2000).

In both conditions, neutral flankers do not refer to any response, while identical and compatible flankers refer to the same response as the target. In line with earlier results, we expected RTs on identical trials to be fastest and RTs on compatible trials to be faster than RTs on neutral trials. However, we did not expect RTs on identical, compatible, and neutral trials to be modulated by social setting, because the flankers on these trials do not refer to a co-actor's response. To test the prediction that the slowing of RTs on incompatible trials relative to the other trial types would be more pronounced in the joint condition than in the individual condition, we averaged RTs across identical, compatible, and neutral trials to obtain a baseline and performed within-subject $2 \times 2$ ANOVAs with the factors Stimulus Type (baseline vs. incompatible) and Condition (individual vs. joint). That is, we compared RTs not predicted to be modulated by Condition (identical, compatible, neutral stimuli) with RTs predicted to be modulated by Condition (incompatible) and tested whether the difference between these two types of trials interacted with Condition (joint vs. individual).

If the slowing of RTs on incompatible trials compared to the baseline was more pronounced in the joint condition compared to the individual condition, a significant interaction between Stimulus Type and Condition should be found. In order to test whether identical, compatible, and neutral trials were affected by social setting, we 
conducted within-subject $2 \times 3$ ANOVAs with the factors Stimulus Type (identical, compatible, neutral) and Condition (individual vs. joint). A main effect of Stimulus Type was expected, with RTs being fastest on identical trials, followed by compatible trials. A main effect of Condition would indicate general differences in speed such as faster performance in the joint condition (social facilitation). A significant two-way interaction would indicate that, contrary to our predictions, social setting differentially affected performance on identical, neutral, and compatible trials.

\section{Experiment 1}

In Experiment 1, participants performed a letter version of the Flanker task (Eriksen and Eriksen 1974). In the joint condition, two participants performed the task with each of them responding to two of four target letters by pressing a single-response key. The exact same go/no-go task was performed without a co-actor in the individual condition. As explained earlier, we predicted a slowing of RTs on incompatible trials compared to the other stimulus types (baseline) selectively in the joint condition. In order to ensure that the material used in the individual and joint conditions yields a standard Flanker effect, we also included a two-choice condition where single participants performed left and right key presses in response to the four target stimuli.

\section{Methods}

\section{Participants}

Forty-eight students (35 women) from Rutgers University, NJ, USA, took part in Experiment 1. They ranged in age from 17 to 39 (mean age 20.1 years). Thirty-two participants performed both the joint and the individual conditions. Sixteen participants performed the two-choice task. All participants received research credits for their participation in partial fulfillment of course requirements. They all had normal or corrected-to-normal vision.

\section{Material and procedure}

Participants were presented with arrays of five letters, with the letter in the middle position constituting the target letter. In the individual and joint conditions, participants responded to two of four target letters. Targets were the letters $\mathrm{H}, \mathrm{K}, \mathrm{S}$, and $\mathrm{C}$, with $\mathrm{H}$ and $\mathrm{K}$ assigned to one key, and $\mathrm{S}$ and $\mathrm{C}$ assigned to the other key. The letters $\mathrm{H}, \mathrm{K}, \mathrm{S}$, $\mathrm{C}$, and $\mathrm{U}$ served as flankers. The combination of these target and flanker letters resulted in four stimulus types: identical, compatible, neutral, and incompatible (see Table 1 for all possible combinations of targets and flankers). Participants always responded with the index finger of the right hand. Exactly the same go/no-go task was performed in the joint and individual conditions, the only difference being that in the joint condition, two participants were sitting side-by-side, whereas in the individual condition, there was an empty chair beside the single participant. Target pairs (H, K vs. S, C), response keys (left vs. right), and the order of conditions (joint vs. individual) were counterbalanced across participants. Each participant responded to the same target pair with the same key throughout the entire experiment.

Each trial started with a fixation cross presented for $500 \mathrm{~ms}$ (centrally positioned, $0.41^{\circ} \times 0.41^{\circ}$ horizontally and vertically), followed by a blank for $500 \mathrm{~ms}$, followed by the stimulus picture (centrally positioned, $1.88^{\circ} \times 0.41^{\circ}$ horizontally and vertically) that was presented for a maximum of 2,000 ms. Once the correct key was pressed, the stimulus picture disappeared from the screen and the next trial started. If a participant committed an error, they received auditory error feedback. If an incorrect response was performed in the joint condition, the stimulus remained on the screen and the correct key remained functional until the time limit of 2,000 ms was reached. Participants received visual error feedback ("too slow") if they took more than $2,000 \mathrm{~ms}$ to respond. No-go stimuli in the individual condition were presented for $800 \mathrm{~ms}$, to roughly adjust the presentation time to the joint condition. After each trial, there was an intertrial interval of $1,000 \mathrm{~ms}$.

Upon arrival at the laboratory, participants were verbally informed that they would be performing two different conditions, acting alone in one condition and taking turns with a second actor in the other condition. At the beginning of each condition, participants were informed about their stimulus-response assignments (Example, joint condition: Person on the LEFT side, press the key in front of you if the middle letter is " $\mathrm{C}$ " or " $\mathrm{S}$ ". Person on the RIGHT side, press the key in front of you if the middle letter is " $\mathrm{H}$ " or "K". Example, individual condition: Please press the key in front of you if the middle letter is "C" or " $\mathrm{S}$ ". Do not respond if the middle letter is " $\mathrm{H}$ " or " $\mathrm{K}$ ".) In both conditions, participants were familiarized with the task (104 trials) before the experiment started. In the main experiment, participants performed three blocks of 96 trials each. All four trial types occurred equally often. At the beginning of each block, participants were reminded of their stimulus response mapping ( $\mathrm{H}, \mathrm{K}$ vs. S, C). In each experimental block, the trials were presented in pseudo-random order.

In the two-choice condition, participants responded to the same stimuli as in the individual and the joint conditions. They were asked to respond to both pairs of target letters with left and right key presses. The combinations of 
target pairs (H, K vs. S, C) and response keys (left vs. right) were counterbalanced across participants.

Results: joint and individual conditions

Only trials in which participants had responded correctly were included in the RT analysis. Errors occurred on 2.5\% of all trials and were not further analyzed. Table 2 shows mean RTs for each condition and each type of stimulus.

A within-subjects $2 \times 2$ ANOVA with the factors Condition (joint vs. individual) and Stimulus Type (baseline vs. incompatible) was computed on RTs. There was a significant main effect for the factor Condition, $F(1$, $31)=5.253, P<.05$, indicating that RTs in the joint condition were faster than RTs in the individual condition. There was also a significant main effect for the factor Stimulus Type, $F(1,31)=97.455, P<.001$, showing that RTs on incompatible trials were slower than baseline RTs. Importantly, there was a significant Condition $\times$ Stimulus Type two-way interaction, $F(1,31)=5.545, P<.05$, demonstrating that the RT difference between incompatible trials and baseline was larger in the joint condition than in the individual condition (see Fig. 2, left side).

Additionally, a within-subjects $2 \times 3$ ANOVA with the factors Condition (joint vs. individual) and Stimulus Type

Table 2 Average RTs (in ms) of Experiments 1-4, for each stimulus type and each condition

\begin{tabular}{|c|c|c|c|c|c|c|c|c|}
\hline \multirow{2}{*}{$\begin{array}{l}\text { Experiment } \\
\text { and condition }\end{array}$} & \multicolumn{2}{|c|}{ Identical } & \multicolumn{2}{|c|}{ Compatible } & \multicolumn{2}{|c|}{ Neutral } & \multicolumn{2}{|c|}{ Incompatible } \\
\hline & $M$ & SD & $M$ & $\mathrm{SD}$ & $M$ & $\mathrm{SD}$ & $M$ & SD \\
\hline \multicolumn{9}{|l|}{ Experiment 1} \\
\hline Two-choice & 611 & 221 & $640 *$ & 232 & 628 & 207 & $697 *$ & 239 \\
\hline Joint & 403 & 109 & $415^{*}$ & 129 & 426 & 118 & $460 *$ & 141 \\
\hline Individual & 429 & 146 & $445^{*}$ & 146 & 451 & 129 & $472 *$ & 137 \\
\hline \multicolumn{9}{|l|}{ Experiment 2} \\
\hline Two-choice & 665 & 246 & 670 & 231 & 664 & 235 & $731^{*}$ & 252 \\
\hline Joint & 474 & 155 & $508^{*}$ & 183 & $472 *$ & 159 & $534 *$ & 208 \\
\hline Individual & 460 & 133 & $488^{*}$ & 150 & 475 & 156 & $498^{*}$ & 148 \\
\hline \multicolumn{9}{|l|}{ Experiment 3} \\
\hline \multicolumn{9}{|l|}{ Order IJ } \\
\hline Joint & 448 & 104 & $463^{*}$ & 104 & $474^{*}$ & 123 & $493^{*}$ & 116 \\
\hline Individual & 453 & 87 & $479^{*}$ & 110 & 479 & 110 & $489^{*}$ & 93 \\
\hline \multicolumn{9}{|l|}{ Order JI } \\
\hline Joint & 434 & 99 & 443 & 92 & 448 & 101 & $475^{*}$ & 130 \\
\hline Individual & 439 & 139 & 449 & 151 & 450 & 105 & $487^{*}$ & 140 \\
\hline \multicolumn{9}{|l|}{ Experiment 4} \\
\hline Intentional & 448 & 121 & $467 *$ & 136 & 467 & 108 & $505 *$ & 174 \\
\hline Unintentional & 474 & 150 & $502 *$ & 175 & 513 & 169 & 512 & 142 \\
\hline
\end{tabular}

An asterisk (*) indicates that the respective RT significantly differs from the RT to its left (identical vs. compatible vs. neutral) was computed on RTs. There were significant main effects for the factors Condition, $F(1,31)=8.266, P<.01$, and Stimulus Type, $F(2,62)=9.651, P<.001$, indicating that RTs in the joint condition were faster than RTs in the individual condition and that RTs differed significantly between the three stimulus types. However, there was no significant Condition $\times$ Stimulus Type two-way interaction, $F(2,62)=$ $0.379, P=.686$.

To determine whether the order in which the individual and joint conditions were performed modulated the results, we conducted a $2 \times 2 \times 2$ ANOVA with the within-subjects factors Condition (joint condition vs. individual condition) and Stimulus Type (baseline vs. incompatible), and the between-subjects factor Order (joint condition first vs. individual condition first). There was no indication that order had any impact on the joint Flanker effect.

Results: two-choice condition

Errors occurred on $3.1 \%$ of all trials and were not further analyzed. Table 2 shows mean RTs for each stimulus type. A paired-samples $t$ test was conducted to compare the means of RTs between incompatible trials (mean: $697 \mathrm{~ms}$ ) and the baseline (mean: $626 \mathrm{~ms}$ ). RTs on incompatible trials were significantly slower, $t(15)=6.314, P<.001$. Additionally, a one-way ANOVA was computed to compare RTs on identical, compatible, and neutral trials. It showed a main effect of Stimulus Type, $F(2,30)=7.13$, $P<.01$. RTs were significantly faster on identical trials compared to compatible trials $(t(15)=4.852, P<.001)$ and neutral trials $(t(15)=2.315, P<.05)$. The difference between neutral and compatible trials was not significant $(t(15)=1.319, P=.27)$.

\section{Discussion}

Pairs of participants performing the Eriksen flanker task together showed a significant Flanker effect (larger RTs on incompatible trials compared to identical, compatible, and neutral trials). Importantly, the Flanker effect was significantly larger in the joint condition compared to the individual condition, where participants performed exactly the same go/no-go task on their own. The most straightforward explanation for the larger RTs on incompatible trials in the joint condition is that increased response interference occurred. We suggest that this interference occurred because each of the co-actors represented their partner's task rules in addition to their own. Therefore, flankers that were part of the co-actor's task (i.e., letters that would require a response by the co-actor if they appeared in the target position) activated a representation 
Fig. 2 Experiment 1 (left) and Experiment 2 (right). Average RTs for identical, neutral, and compatible trials (baseline; white bars) and incompatible trials (black bars), in the joint and in the individual condition

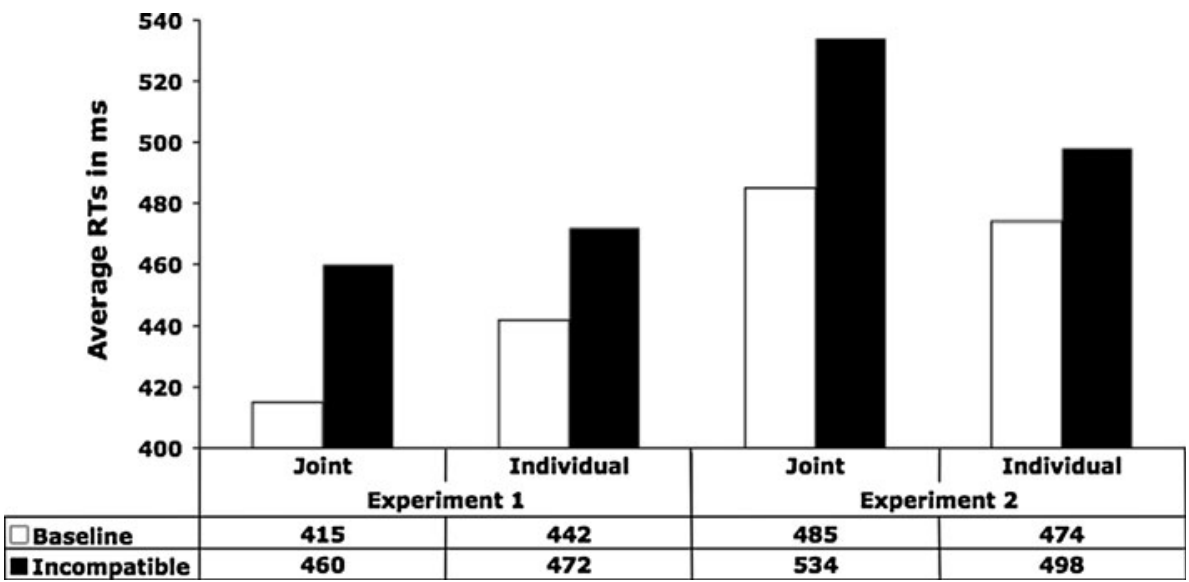

of the co-actor's action alternative, interfering with the selection of one's own response.

As expected, the RT pattern of identical, compatible, and neutral trials was not modulated by Condition. However, reaction times were generally faster in the joint condition than in the individual condition. This finding is most likely due to social facilitation, the phenomenon that people tend to act faster in the presence of another person, especially when the task is relatively easy (e.g., Aiello and Douthitt 2001; Guerin 1993; Zajonc 1965). This overall RT difference cannot explain why the difference between incompatible trials and baseline (average of identical, compatible, neutral trials) was larger in the joint condition than in the individual condition.

A significant Flanker effect was observed not only in the joint condition but also in the individual condition (see mean RTs in Table 2), as predicted by the dimensional overlap model (Kornblum and Lee 1995). Incompatible flankers appeared as targets on no-go trials, whereas neutral flankers never appeared in the target position. Accordingly, responses to incompatible flankers needed to be inhibited when these appeared in the target position on no-go trials, whereas neutral flankers never required inhibition. Similar go/no-go Flanker effects have been found in previous studies (Heil et al. 2000; Kopp et al. 1996).

Finally, the RT pattern obtained in the two-choice condition showed the standard pattern of results observed in studies on the Flanker effect. The lack of a difference between RTs on neutral trials and RTs on compatible trials is not uncommon. Typically, RTs on compatible trials are slightly faster than RTs on neutral trials (Sanders and Lamers 2002) but similar RTs for neutral and compatible trials have been reported. For instance, Hazeltine et al. (2003) found no difference between neutral and compatible trials in a letter version of the Flanker task as well as in a color version. Whereas incompatible flankers reliably increase RTs, compatible flankers are known to produce less-consistent results (Madden and Langley 2003). In particular, it has been proposed that compatible flankers may lead to a competition in the recognition of target and flankers and thereby disrupt performance (Eriksen and Schultz 1979; Grice and Gwynne 1985; Proctor and Fober 1985). Apparently, response interference dominated over response facilitation in the present version of the task.

\section{Experiment 2}

It has been proposed that there are different components of response selection in the Eriksen flanker task, some of which depend on the stimulus material, whereas others are independent of the stimulus material (Hazeltine et al. 2003). Comparing a letter and a color version of an Eriksen flanker task, Hazeltine et al. found that different areas in prefrontal cortex were active depending on the type of stimulus information that needed to be inhibited. The aim of Experiment 2 was to test whether the joint Flanker effect can be generalized to a different set of stimuli. This would indicate that response interference on incompatible trials in the joint condition occurs independently of the stimulus material. Participants performed a color version of the Flanker task (Hazeltine et al. 2000), following the exact same procedure as in Experiment 1. In the color version, participants saw three colored circles and were asked to respond to the color of the middle circle. We predicted that the slowing of RTs on incompatible trials compared to all other stimulus types (baseline) would be more pronounced in the joint condition than in the individual condition.

\section{Method}

\section{Participants}

Thirty-one students (15 women) from Rutgers University, NJ, USA, took part in Experiment 2. They ranged in age 
from 16 to 50 (mean age 20.4 years). Sixteen participants performed both the joint and the individual conditions. Fifteen participants performed the two-choice task. All participants received research credits for their participation. They all had normal or corrected-to-normal vision.

\section{Material and procedure}

Stimuli consisted of three colored circles with the middle circle constituting the target. Each participant responded to two of four target colors. Red and purple were assigned to one key, blue and green to the other key. The colors red, purple, blue, green, and gray served as flankers. In order to enhance visual contrast, the colored circles (centrally positioned, $1.31^{\circ} \times 0.25^{\circ}$ horizontally and vertically) were surrounded by a black rectangle (centrally positioned, $5.31^{\circ} \times 3.6^{\circ}$ horizontally and vertically), while the rest of the screen was white. After practice ( 88 trials), participants performed three blocks of 80 trials each. Apart from these changes, the procedure was exactly the same as in Experiment 1 .

Results: joint and individual conditions

Errors occurred on $1.2 \%$ of all trials. Only trials in which participants had responded correctly were included in the RT analysis. Table 2 shows mean RTs for each condition and each stimulus type.

A within-subjects $2 \times 2$ ANOVA with the factors Condition (joint vs. individual) and Stimulus Type (baseline vs. incompatible) showed a significant main effect of Stimulus Type, $F(3,15)=22.595, P<.001$. RTs on incompatible trials were larger than baseline RTs. Furthermore, there was a significant Condition $\times$ Stimulus Type two-way interaction, $F(1,15)=16.169, P<.01$, demonstrating that the RT difference between incompatible trials and baseline was larger in the joint condition than in the individual condition (see Fig. 2, right side). There was no significant main effect of Condition, $F(1,15)=2.219$, $P=157$.

A within-subjects $2 \times 3$ ANOVA with the factors Condition (joint condition vs. individual condition) and Stimulus Type (identical vs. compatible vs. neutral) revealed a significant main effect of Stimulus Type $(F(2$, $30)=13.338, P<.001)$, indicating that overall RTs differed between types of stimuli. There were no further significant main effects or interactions.

A $2 \times 2 \times 2$ ANOVA with the within-subjects factors Condition (joint condition vs. individual condition) and Stimulus Type (baseline vs. incompatible), and the between-subjects factor Order (joint condition first vs. individual condition first) provided no indication for order of conditions modulating the joint Flanker effect.
Results: two-choice condition

Errors occurred on $3.7 \%$ of all trials and were not further analyzed. Table 2 shows mean RTs for each stimulus type. A paired-samples $t$ test revealed a significant difference between stimulus types (baseline vs. incompatible), $t(14)=3.592, P<.01$. RTs on incompatible trials (mean: $731 \mathrm{~ms}$ ) were larger than baseline RTs (mean: $666 \mathrm{~ms}$ ). A one-way ANOVA comparing RTs on identical, compatible, and neutral trials did not show a significant main effect of timulus type, $F(2,28)=.413, P=.665$. Accordingly, two-sided $t$ tests did not show a significant difference between identical, compatible, and neutral trials (all $P>$.05).

\section{Discussion}

As in Experiment 1, the slowing of RTs on incompatible trials compared to all other stimulus types (baseline) was larger in the joint condition than in the individual condition. This provides further evidence for the assumption that co-actors represented not only the task rules according to which they acted, but also the task rules according to which their partner acted.

In contrast to Experiment 1, no effects of social facilitation were observed. This could be due to stimulus differences or differences in the sample. Importantly, this demonstrates that the interaction between Condition and Stimulus Type does not hinge on a general speed difference between the individual and joint conditions.

Unexpectedly, RTs were notably fast on neutral trials in all conditions. Possibly, the gray stimuli used as neutral flankers created a particularly salient contrast to the other colors and thus facilitated perceptual processing (see Eriksen and Eriksen 1974 for similarity manipulations of neutral flankers). Since this facilitation on neutral flankers occurred across all conditions, the interpretation of the differences between the joint and individual conditions is not affected.

As expected, the two-choice task also showed a significant flanker effect. The lack of a difference between identical and compatible trials in this condition is likely due to the particular stimulus material used (Cohen and Shoup 1997) and has been found in previous studies (e.g., Flowers 1990; Miller 1991).

\section{Experiment 3}

Experiments 1 and 2 provide evidence that co-actors represent each other's stimulus-response mappings. However, it is unclear whether knowledge about the co-actor's task rules is sufficient for the joint Flanker effect to occur, or 
whether observing one's partner's actions is necessary. Previous studies on joint performance of a spatial compatibility task suggest that believing to be acting together with another individual is sufficient (Ruys and Aarts 2010; Sebanz et al. 2005; Tsai et al. 2008; Vlainic et al. 2010). However, in these studies, the links between stimuli and responses were automatic, and thus, the stimuli may have contributed to keeping a representation of the co-actor's response alternative active. Do people take into account the task rules of an invisible co-actor even when they both act according to arbitrary S-R mappings?

To address this question, participants in Experiment 3 were instructed to perform the Flanker task with a co-actor seated in another room. In fact, participants never interacted with another person, but performed the task by themselves, either believing that they were performing the task with a co-actor or believing that they were performing the task on their own (as in Tsai et al. 2008).

\section{Method}

\section{Participants}

Fifty-seven students (33 women) from Rutgers University, NJ, USA, took part in Experiment 3, performing both the individual and the 'fake' joint condition. Participants ranged in age from 17 to 39 (mean age 20.8 years). All participants received research credits for their participation. They all had normal or corrected-to-normal vision.

\section{Material and procedure}

Stimuli were the same as in Experiment 1. In both conditions, there was only one response key located in front of the participant. Participants were seated centrally in front of the computer screen. There was no empty chair next to the participant.

The course of each trial was the same as in Experiment 1, with the following exceptions: The fixation cross (centrally positioned) measured $0.25^{\circ} \times 0.25^{\circ}$ horizontally and vertically, and the stimulus picture (centrally positioned) measured $1.7^{\circ} \times 0.4^{\circ}$ horizontally and vertically. Participants received feedback in the form of a green check mark if they had responded within the 2,000-ms time window on go trials. In the joint condition, participants saw the same check mark after every no-go trial, to maintain the belief that a co-actor was taking care of the other half of the task. The green check was presented for $500 \mathrm{~ms}$. After an ITI of $500 \mathrm{~ms}$, the next trial started. No-go stimuli in both conditions were presented for either 400, 500, 600, 700, or $800 \mathrm{~ms}$, to roughly adjust the presentation time to the RTs of a real co-actor. These presentation times were equally frequent and occurred in randomized order across consecutive trials.

As in Experiment 1 and 2, once participants arrived at the laboratory, they were verbally informed that they would be performing two different conditions, acting alone in one condition and together in the other condition. In the joint condition, the experimenter told the participants that they would be performing the task together with her, but sitting in different rooms. The experimenter said she would be sitting in the room opposite of the testing room, without specifying her exact sitting position. In order to foster the belief that the participant was interacting with the experimenter in another room, the following manipulation was built into the joint condition. After the experimenter had left, participants were asked to start the experiment by pressing the space bar. After the space bar was pressed, there was a blank $(6,000 \mathrm{~ms}$ in the training phase, $4,000 \mathrm{~ms}$ in block one, 5,000 ms in block two, 3,000 ms in block three, and 6,000 $\mathrm{ms}$ in block four) preceding the following message: "Both persons are now ready to start the experiment. The training phase/block $\mathrm{X}_{(1,2,3)}$ will start automatically in $10 \mathrm{~s}$ ", which was presented for $10,000 \mathrm{~ms}$. Then, the first trial started. In both conditions, participants were first familiarized with the task ( 88 trials), and then performed three blocks of 80 trials each. After the experiment, participants were asked whether they indeed believed that they had performed the task alone in the individual condition and together with the experimenter in the joint condition, and were subsequently debriefed.

\section{Results}

Seventeen of the fifty-seven participants reported suspecting that the experimenter was not performing the task in the other room (joint condition) or that they were not performing the task alone (individual condition). These participants were removed from further analyses. Note that this relatively high number is due to our strict exclusion criterion; we excluded every participant who voiced any doubt that the experimenter had performed her task in the other room to make sure that our findings reflect the performance of participants who were truly convinced that they were performing the task with a co-actor.

Errors occurred on $0.9 \%$ of the trials. Only trials in which participants had responded correctly were included in RT analysis. Table 2 shows mean RTs for each condition and each stimulus type.

A within-subjects $2 \times 2$ ANOVA with the factors Condition (joint condition vs. individual condition) and Stimulus Type (baseline vs. incompatible) was computed. There was a significant main effect of Stimulus Type, $F(1,39)=94.198$, $P<.001$, showing that RTs on incompatible trials were larger than baseline RTs (identical, compatible, and neutral). 
Against our expectations, there was no significant Condition $\times$ Stimulus Type two-way interaction, $F(1,39)=0.396$, $P=.533$, indicating that the size of the Flanker effect was not modulated by Condition.

A within-subjects $2 \times 3$ ANOVA with the factors Condition (joint condition vs. individual condition) and Stimulus Type (identical vs. compatible vs. neutral) revealed a significant main effect of Stimulus Type $(F(2$, $112)=33.790, P<$.001). There were no further significant main effects or interactions.

To determine whether the order in which the individual and joint conditions had been performed modulated the results, we conducted a $2 \times 2 \times 2$ ANOVA with the within-subjects factors Condition (joint vs. individual) and Stimulus Type (baseline vs. incompatible), and the between-subjects factor Order (joint condition first vs. individual condition first). There was a significant Condition $\times$ Stimulus Type $\times$ Order three-way interaction, $F(1$, $38)=6.419, P<.05$. The difference between incompatible trials and baseline was larger in the joint condition than in the individual condition, but only when the individual condition had been performed first (see Fig. 3, right side). None of the other effects were significant.

In a next step, we analyzed the results of the group that had performed the individual condition first separately. A $2 \times 2$ ANOVA with the within-subjects factors Condition (joint vs. individual) and Stimulus Type (baseline vs. incompatible) showed a significant main effect for the factor Stimulus Type, $F(1,19)=55.912, P<.001$, as well as the predicted Condition $\times$ Stimulus Type two-way interaction, $F(1,19)=5.623, P<.05$.

Additionally, a $2 \times 3 \times 2$ ANOVA with the withinsubjects factors Condition (joint condition vs. individual condition) and Stimulus Type (identical vs. compatible vs. neutral), and the between-subjects factor Order (joint

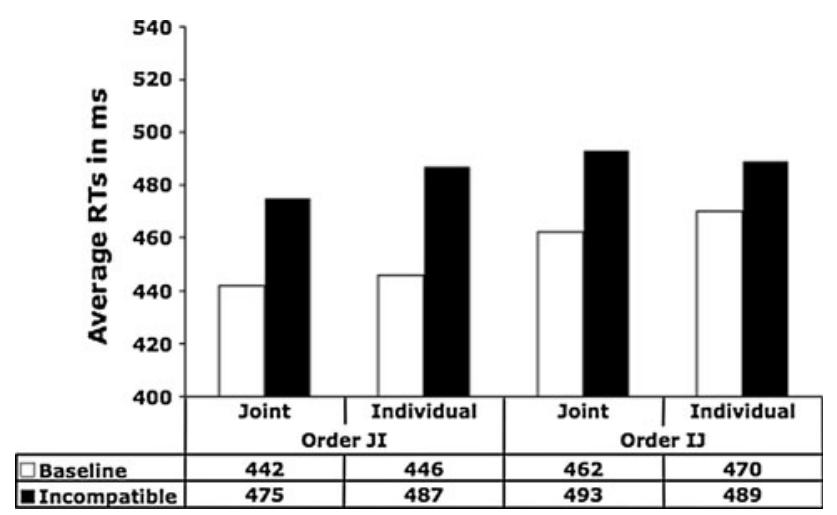

Fig. 3 Average RTs for identical, neutral, and compatible trials (baseline; white bars) and incompatible trials (black bars) in the joint and in the individual condition. Left results from participants who performed the joint condition prior to the individual condition. Right results from participants who performed the individual condition prior to the joint condition condition first vs. individual condition first) was computed on RTs. There was a significant main effect for the factor Stimulus Type, $F(2,76)=21.43, P<.001$. There were no further significant main effects or interactions.

\section{Discussion}

Overall, Experiment 3 did not show the same pattern of results as the previous experiments. When participants believed to be interacting with another person, the difference between incompatible trials and baseline was similar in the joint and individual conditions. However, a closer look at the data revealed that the order in which participants completed the two conditions modulated the results. When the individual condition preceded the joint condition, the findings were the same as in the two previous experiments. In contrast, when the joint condition preceded the individual condition, there was a large difference between incompatible trials and baseline in both conditions.

In our view, the most likely explanation for this pattern is that participants who performed the individual condition after the joint condition may have been unable to shed the representation of the other actor and/or their task rule once the task had been encoded as a joint one. That is, once participants believed they had performed the task with another actor, they kept thinking of the other actor and/or their task even when acting alone. This interpretation is supported by the finding that the Flanker effect in the joint condition did not differ depending on Order $(t(38)=0.303$; $P=.764)$, while the Flanker effect in the individual condition did $(t(38)=2.914 ; P<.01)$. This indicates that, while task co-representation occurred in the joint condition irrespective of Order, participants represented the other task in the individual condition when they had first executed the joint condition. Furthermore, a comparison with Experiment 1 showed a significant Condition (joint vs. individual) $\times$ Stimulus Type (baseline vs. incompatible) $\times$ Order (joint condition first vs. individual condition first $) \times$ Experiment (Experiment 1 vs. Experiment 3$)$ four-way interaction $(F(1$, $68)=7.638, P<.01)$, indicating that the joint Flanker effect differed between the two experiments, depending on the order in which conditions were conducted. However, there was no significant Condition $\times$ Stimulus Type $\times$ Experiment three-way interaction $(F(1,68)=2.974$, $P=.089$ ). That is, the overall joint Flanker effect was not modulated by experimental setting, suggesting that the observed order effect in Experiment 3 was related to the belief manipulation. Beliefs may be harder to switch on and off compared to having direct evidence about the presence or absence of one's co-actor. Taken together, the results of Experiment 3 are in line with earlier studies showing that the mere belief to be acting together with another person can be sufficient for task co-representation to occur, 
generalizing this finding to a situation where co-actors perform tasks with arbitrary S-R mappings.

\section{Experiment 4}

In Experiment 4, we addressed a further boundary condition for task co-representation. Previous research by Tsai et al. (2008) indicated that the intentionality of the co-actor may matter. A joint Simon effect was observed when participants believed that another person in a different room performed a complementary go/no-go task, but not when they believed that a computer took care of the same go/no-go task. However, since humans and computers differ in so many ways, one cannot be sure that the co-actor's intentionality was the key factor. It remains unclear how participants represented the computer-as an unintentional agent, as a nonbiological agent, or just as the nonexistence of an agent, to name just a few possibilities. To test whether task co-representation occurs specifically when people perceive a co-actor as acting intentionally, we compared performance when participants acted next to a co-actor acting intentionally and when they acted next to the same co-actor, but her actions were controlled by a machine.

Participants performed the joint Flanker task (letter version) twice with the same confederate. In the "intentional co-actor" condition, the confederate performed her task by actively pressing a key when it was her turn. In the "unintentional co-actor" condition, the confederate's finger was pulled down by an electromagnet each time it was her turn to act (see Fig. 4, bottom). This ensured that the two conditions were identical but for the cause of the confederate's actions. We predicted a larger joint Flanker effect in the intentional compared to the unintentional coactor condition.

\section{Method}

\section{Participants}

Twenty students (15 women) from Rutgers University, NJ, USA, took part in the experiment. Participants ranged in age from 18 to 35 (mean age 20.8 years). All participants received research credits for their participation. They all had normal or corrected-to-normal vision.

\section{Material and procedure}

Participants performed both conditions with the same confederate. In the intentional co-actor condition, the confederate was actively performing her part of the Flanker task. In the unintentional co-actor condition, the confederate was wearing an iron ring on her right index finger. On

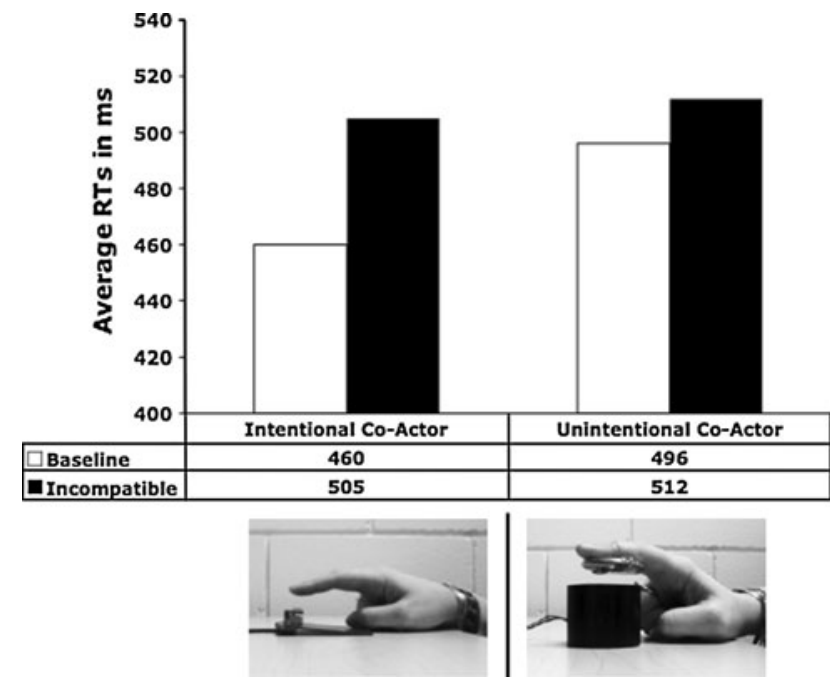

Fig. 4 Average RTs for identical, neutral, and compatible trials (baseline; white bars) and incompatible trials (black bars) in the intentional co-actor condition (left) and in the unintentional co-actor condition (right)

trials requiring her response, the computer activated the electromagnet, which attracted the iron ring held closely above the magnet. This resulted in the confederate's finger being pulled down to hit the magnet (see Fig. 4, bottom). Participants were told that the magnet functioned like a response key and that a response was recorded the moment the finger touched the magnet. Participants had the opportunity to try out the electromagnet and went through a training block before the experiment started.

Stimuli and the course of each trial were the same as in Experiment 1 with the following exceptions. Stimuli the confederate passively responded to in the unintentional coactor condition were presented for $800 \mathrm{~ms}$, to roughly adjust the presentation time to the intentional co-actor condition. After $600 \mathrm{~ms}$, the electromagnet was activated, and the force of the magnet pulled down the confederate's finger. The magnet was deactivated at the end of stimulus presentation.

Target stimuli and the position of response keys were counterbalanced across participants. All participants performed the unintentional co-actor condition first. This was to avoid that once participants perceived the co-actor as acting intentionally, they would continue to ascribe intentionality to her actions in the unintentional co-actor condition.

Results

Incorrect responses (1.6\%) were removed from RT analyses. Table 2 shows mean RTs for each condition and each stimulus type.

A $2 \times 2$ ANOVA with the within-subjects factors Condition (intentional co-actor vs. unintentional co-actor) 
and Stimulus Type (baseline vs. incompatible) revealed a significant main effect of Stimulus Type $(F(1,19)=$ $21.133, P<.001)$ and a significant Condition $\times$ Stimulus Type two-way interaction $(F(1,19)=10.277, P<.01)$. As predicted, the difference between incompatible trials and baseline was significantly larger in the intentional co-actor condition (see Fig. 4). There was a tendency for a main effect of Condition $(F(1,19)=3.395, P=.081)$. Overall RTs tended to be faster in the intentional compared to the unintentional co-actor condition.

To compare the Flanker effect in the unintentional condition with that of the individual condition of Experiment 1 , we conducted a $2 \times 2$ ANOVA with the betweensubject factor Experiment 1 (1 vs. 4) and the within-subject factor Trial Type (baseline vs. incompatible). To make sure that the data are comparable, we only included data from participants in Experiment 1 who had performed the individual condition first (because all participants in Experiment 4 performed the unintentional condition first). There was no significant interaction, $F(1,50)=1.238, P=.274$, supporting the conclusion that the co-actor's task was not represented in the unintentional condition.

A further $2 \times 3$ ANOVA with the within-subjects factors Condition (intentional co-actor vs. unintentional coactor) and Stimulus Type (identical vs. compatible vs. neutral) revealed significant main effects for the factors Condition $(F(1,19)=9.559, P<.01)$ and Stimulus Type $(F(2,38)=12.029, P<.001)$. RTs were significantly faster when acting next to an intentional co-actor. There was no significant interaction.

\section{Discussion}

The results of Experiment 4 show that stronger response interference on incompatible trials occurred when the coactor acted intentionally compared to when her actions were externally controlled. This provides clear evidence that the perceived intentionality of a co-actor modulates task corepresentation (Tsai et al. 2008). RTs were overall faster in the intentional co-actor condition. This may either reflect social facilitation effects or a slowing of responses in the unintentional co-actor condition induced by the relatively slow responses of the co-actor. However, the overall RT difference cannot explain why the difference between incompatible trials and other stimulus types (identical, compatible, neutral) was larger when the co-actor acted intentionally.

\section{General discussion}

The aim of the present study was to test whether co-actors take into account the task rules governing each other's performance in a paradigm where the links between stimuli and responses are arbitrary. Using different stimulus sets (letters and colors), Experiments 1 and 2 provided converging evidence that co-actors represented each other's task rules. Specifically, participants responded slower to the same stimuli when the flankers referred to the coactor's task, compared to when the flankers referred to their own task or did not refer to any task. This joint Flanker effect suggests that when two people perform independent tasks next to each other, they keep in mind both task rules-their own and the co-actor's. This extends earlier studies on joint task performance that focused on tasks including automatic stimulus-response links. The present findings demonstrate that stimuli pertaining to a co-actor's task activate representations of the co-actor's actions even when the links between stimuli and responses are arbitrary.

The results of Experiment 3 provided evidence that believing to be performing the Flanker task together with another person can be sufficient to elicit a joint Flanker effect. Whereas results from variants of the joint Simon task indicate that co-representation effects involve spatial coding of one's responses relative to the co-actor's (Guagnano et al. 2010; Milanese et al. in press), the results of Experiment 3 demonstrate that, for cognitive paradigms using arbitrary S-R mappings, task co-representation can also occur when the precise spatial position of a co-actor relative to oneself is unknown and all that is known is the other's S-R mapping. In addition, the results suggest that once participants had represented the other's task, they retained this task set even when they were led to believe that they were now on their own. It almost seemed as if they could not get rid of the imagined co-actor in their mind.

Experiment 4 demonstrated that task co-representation depends on perceiving the co-actor as performing her task intentionally. Participants showed a joint Flanker effect when performing the task with a co-actor in control of her actions, but not when they performed the same task with a co-actor whose actions were controlled by an electromagnet. Whereas previous studies left open why inanimate agents' tasks were ignored, the present results point to the co-actor's intentionality as a key factor. Our results provide clear evidence that human sensitivity to intentional action, which already manifests itself in infancy (e.g., Sommerville and Woodward 2005), leads us to share others' tasks only when we think that they act by their own will.

The standard Flanker effect is assumed to arise at the level of response selection, because target and flankers activate two different responses (Eriksen and Schultz 1979; Grice and Gwynne 1985). The joint Flanker effect can be explained in a similar way. As each participant forms a representation of the co-actor's S-R mappings, incompatible flankers activate a representation of the co-actor's 
response. Accordingly, the slower RTs on incompatible trials would reflect a response selection conflict.

It is possible, however, that the slowing of RTs on incompatible trials in the joint condition does not reflect interference between two competing response activations but instead is due to increased response inhibition triggered by the flankers. EEG studies using the joint Simon task have found evidence for increased inhibition on no-go trials where the co-actor needed to respond, compared to individual no-go trials (Sebanz et al. 2006; Tsai et al. 2008). It could be that no-go stimuli in the joint condition of the Flanker task become associated with increased inhibition, so that when these stimuli are flankers (incompatible go trials), participants need to overcome the tendency to inhibit their actions. As more inhibition occurs on joint no-go trials, more conflict occurs on incompatible trials in the joint condition compared to the individual condition.

Furthermore, participants in the joint condition may have represented the whole set of task rules in a qualitatively different way. In particular, they may have reformulated the task rules to distinguish between stimuli requiring "my response" and stimuli requiring "your response". Stimuli that required "your response" surrounding a stimulus requiring "my response" (incompatible stimuli) may have created a turn-taking conflict because people needed to figure out whose turn it was (Philipp and Prinz 2010). In support of this view, an fMRI study, contrasting joint and individual performance of a go/no-go task (Sebanz et al. 2007), found that in the joint condition, there was higher activation in areas involved in self-other distinction (e.g., Brass et al. 2005) and increased self-awareness (e.g., Mitchell et al. 2006). This could be interpreted as evidence that people form a hierarchical task representation in social situations with self/other on top (Roepstorff and Frith 2004), and the particular tasks and actions represented lower down in the hierarchy.

Needless to say, many questions about the boundary conditions of task co-representation remain. How cognitively demanding can each individual's task be for task corepresentation to still occur? For example, does the complexity of the S-R mapping at hand or the number of response alternatives determine whether shared task representations are formed? Do participants also co-represent $\mathrm{S}-\mathrm{R}$ mappings if there is more than one co-actor? Is working memory load a limiting factor, and if so, can this limit be expanded by introducing a situation where the coactors have an explicit advantage of taking each other into account? These and many more questions need to be addressed in future studies.

Selectively sharing tasks with intentional actors may be functional both in terms of collaboration as well as in terms of competition. It is crucial for cooperation because it allows one to perform different parts of a joint task (Newman-Norlund et al. 2007), to predict others' actions (Knoblich and Jordan 2003; Sebanz and Knoblich 2009), and to monitor their errors (van Schie et al. 2004). More generally, it may reflect humans' unique tendency toward joint commitments (Warneken and Tomasello 2006). However, keeping others' tasks in mind may also be helpful for outperforming others in competitive situations (e.g., De Bruijn et al. 2008; Ruys and Aarts 2010).

For now, we would like to conclude that people have a remarkable tendency to take into account what others are doing even when this is not required. This tendency may have its origins in requirements of joint action. Many forms of joint action require that people anticipate what others are going to do next. Clearly, representing others' tasks provides a powerful means for predicting their actions in addition to observing what they are doing. Most importantly, it allows individuals to rely on cues in the environment to know what others are likely going to do, thus creating a type of common ground (Clark 1996) that is not necessarily bound to language. A challenge for future work will be to clarify how task co-representation works in concert with more fine-grained predictive mechanisms that are crucial for the temporal coordination of actions and higher-level mechanisms employed in more sophisticated forms of social interactions.

Acknowledgments We would like to thank Andrea Philipp and Sandro Rubicchi for their comments on an earlier version of this manuscript. We also thank Antje Holländer, Dorit Wenke, Felix Warneken, Wolfgang Prinz, Thomas Dolk, Roman Liepelt, Jasmin Steinwender, and Katharina Hamann for discussions and comments, and we thank Katya Dobryakova Kravtsov for her help in data collection.

Open Access This article is distributed under the terms of the Creative Commons Attribution Noncommercial License which permits any noncommercial use, distribution, and reproduction in any medium, provided the original author(s) and source are credited.

\section{References}

Aiello JR, Douthitt EA (2001) Social facilitation from Triplett to electronic performance monitoring. Group Dyn 5:163-180

Amazeen PG, Schmidt RC, Turvey MT (1995) Frequency detuning of the phase entrainment dynamics of visually coupled rhythmic movements. Biol Cybern 72:511-518

Atmaca S, Sebanz N, Prinz W, Knoblich G (2008) Action corepresentation: the joint SNARC effect. Soc Neurosci 3:1-11

Botvinick MM, Braver TS, Carter CS, Cohen JD (2001) Conflict monitoring and cognitive control. Psychol Rev 108:624-652

Brass M, Bekkering H, Wohlschläger A, Prinz W (2000) Compatibility between observed and executed finger movements: comparing symbolic, spatial, and imitative cues. Brain Cogn 44:124-143 
Brass M, Bekkering H, Prinz W (2001) Movement observation affects movement execution in a simple response task. Acta Psychol 106:3-22

Brass M, Derfuss J, von Cramon DY (2005) The inhibition of imitative and overlearned responses: a functional double dissociation. Neuropsychologia 43:89-98

Clark HH (1996) Using language. Cambridge University Press, Cambridge

Cohen A, Shoup R (1997) Perceptual dimensional constraints in response selection processes. Cogn Psychol 32:128-181

Coles MGH, Gratton G, Bashore TR, Eriksen CW, Donchin E (1985) A psychophysiological investigation of the continuous flow model of human information processing. J Exp Psychol Hum Percept Perform 11:529-553

De Bruijn ER, Miedl SF, Bekkering H (2008) Fast responders have blinders on: ERP correlates of response inhibition in competition. Cortex 44:580-586

Dehaene S, Bossini S, Giraux P (1993) The mental representation of parity and number magnitude. J Exp Psychol Gen 122:371-396

Eriksen BA, Eriksen CW (1974) Effects of noise letters upon the identification of a target letter in a nonsearch task. Percept Psychophys 16:143-149

Eriksen CW, Eriksen BA (1979) Target redundancy in visual search: do repetitions of the target within the display impair processing? Percept Psychophys 26:195-205

Eriksen CW, Schultz DW (1979) Information processing in visual search: a continuous flow conception and experimental results. Percept Psychophys 25:249-263

Flowers JH (1990) Priming effects in perceptual classification. Percept Psychophys 47:135-148

Grice RG, Gwynne JW (1985) Temporal characteristics of noise conditions producing facilitation and interference. Percept Psychophys 37:495-501

Guagnano D, Rusconi E, Umilta C (2010) Sharing a task or sharing space? On the effect of the confederate in action coding in a detection task. Cognition 114:348-355

Guerin B (1993) Social facilitation. Cambridge University Press, Cambridge

Hazeltine E, Poldrack R, Gabrieli JDE (2000) Neural activation during response competition. J Cogn Neurosci 12:118-129

Hazeltine E, Bunge SA, Scanlon MD, Gabrieli JDE (2003) Materialdependent and material-independent selection processes in the frontal and parietal lobes: an event-related fMRI investigation of response competition. Neuropsychologia 41:1208-1217

Heil M, Osman A, Wiegelmann J, Rolke B, Hennighausen E (2000) N200 in the Eriksen-task: inhibitory executive processes? J Psychophysiol 14:218-225

Kilner JM, Paulignan Y, Blakemore SJ (2003) An interference effect of observed biological movement on action. Curr Biol 13:522-525

Knoblich G, Jordan S (2003) Action coordination in individuals and groups: learning anticipatory control. J Exp Psychol Learn Mem Cogn 29:1006-1016

Kopp B, Rist F, Mattler U (1996) N200 in the flanker task as a neurobehavioral tool for investigating executive control. Psychophys 33:282-294

Kornblum S, Lee JW (1995) Stimulus-response compatibility with relevant and irrelevant stimulus dimensions that do and do not overlap with the response. J Exp Psychol Hum Percept Perform $21: 855-875$

Kornblum S, Hasbroucq T, Osman A (1990) Dimensional overlap: cognitive basis for stimulus-response compatibility-a model and taxonomy. Psychol Res 97:253-270

Longo M, Kosobud A, Bertenthal B (2008) Automatic imitation of biomechanically possible and impossible actions: effects of priming movements vs. goals. J Exp Psychol Hum Percept Perform 34:489-501
Madden DJ, Langley LK (2003) Age-related changes in selective attention and perceptual load during visual search. Psychol Aging 18:54-67

Marsh KL, Richardson MJ, Schmidt RC (2009) Social connection through joint action and interpersonal coordination. TopiCS $1: 320-339$

Milanese N, Iani C, Rubichi S (2010) Shared learning shapes human performance: transfer effects in task sharing. Cognition 116:15-22

Milanese N, Iani C, Sebanz N, Rubichi S (in press) Contextual determinants of the social transfer of learning effect. Exp Brain Res

Miller S (1988) Joint action: the individual strikes back. In: Tsohatzidis SL (ed) Intentional acts and institutional facts: essays on john searle's social ontology. Springer Press, Dordrecht

Miller J (1991) The flanker compatibility effect as a function of visual angle, attentional focus, visual transients, and perceptual load: a search for boundary conditions. Percept Psychophys 49:270-288

Mitchell JP, Macrae CN, Banaji MR (2006) Dissociable medial prefrontal contributions to judgments of similar and dissimilar others. Neuron 50:655-663

Newman-Norlund RD, van Schie HT, van Zuijlen AMJ, Bekkering H (2007) The mirror neuron system is more active during complementary compared with imitative action. Nat Neurosci 10:817-818

Philipp AM, Prinz W (2010) Evidence for a role of the responding agent in the joint compatibility effect. Q J Exp Psychol 63:2159-2171

Prinz W (1997) Perception and action planning. Eur J Cog Psychol 9:129-154

Proctor RW, Fober GW (1985) Repeated-stimulus superiority and inferiority effects in the identification of letters and digits. Percept Psychophys 38:125-134

Richardson MJ, Marsh KL, Schmidt RC (2005) Effects of visual and verbal interaction on unintentional interpersonal coordination. J Exp Psychol Hum Percept Perform 31:62-79

Richardson MJ, Marsh KL, Isenhower R, Goodman J, Schmidt RC (2007) Rocking together: dynamics of intentional and unintentional interpersonal coordination. Hum Mov Sci 26:867-891

Rizzolatti G, Sinigaglia C (2010) The functional role of the parietofrontal mirror circuit: interpretations and misinterpretations. Nat Rev Neurosci 11:264-274

Roepstorff A, Frith C (2004) What's at the top in the top-down control of action? Script-sharing and 'top-top' control of action in cognitive experiments. Psychol Res 68:189-198

Ruys KI, Aarts H (2010) When competition merges people's behavior: interdependency activates shared action representations. J Exp Soc Psychol 46:1130-1133

Sanders AF, Lamers JM (2002) The Erisksen flanker effect revisited. Acta Psychol 109:41-56

Schmidt RC, Bienvenu M, Fitzpatrick P, Amazeen P (1998) A comparison of within- and between-person coordination: coordination breakdown and coupling strength. J Exp Psychol Hum Percept Perform 24:884-890

Sebanz N, Knoblich G (2009) Jumping on the ecological bandwagon? Mind the gap!. Eur J Soc Psychol 39:1230-1233

Sebanz N, Knoblich G, Prinz W (2003) Representing others' actions: just like one's own? Cognition 88:B11-B21

Sebanz N, Knoblich G, Prinz W (2005) How to share a task: corepresenting stimulus-response mappings. J Exp Psychol Hum Percept Perform 33:1234-1246

Sebanz N, Knoblich G, Prinz W, Wascher E (2006) Twin peaks: an ERP study of action planning and control in coacting individuals. J Cogn Neurosci 18:859-870

Sebanz N, Rebbechi D, Knoblich G, Prinz W, Frith C (2007) Is it really my turn? An event-related fMRI study of task sharing. Soc Neurosci 2:81-95 
Simon JR (1990) The effects of an irrelevant directional cue on human information processing. In: Proctor RW, Reeve TG (eds) Stimulus-response compatibility: an integrated perspective. North-Holland, Amsterdam

Sommerville JA, Woodward AL (2005) Pulling out the intentional structure of action: the relation between action processing and action production in infancy. Cognition 95:1-30

Stuermer B, Aschersleben G, Prinz W (2000) Correspondence effects with manual gestures and postures: a study of imitation. J Exp Psychol Hum Percept Perform 26:1746-1759

Tsai CC, Kuo WJ, Hung DL, Tzeng OJL (2008) Action co-representation is tuned to other humans. J Cogn Neurosci 20:2015-2024

Van Schie HT, Mars RB, Coles MGH, Bekkering H (2004) Modulation of activity in medial frontal and motor cortices during error observation. Nat Neurosci 7:549-554

Van Ulzen NR, Lamoth CJ, Daffertshofer A, Semin GR, Beek PJ (2008) Characteristics of instructed and uninstructed interpersonal coordination while walking in pairs. Neurosci Lett 432:88-93
Van Veen V, Cohen JD, Botvinick MM, Stenger VA, Carter CS (2001) Anterior cingulate cortex, conflict monitoring, and levels of processing. Neuroimage 14:1302-1308

Vlainic E, Liepelt R, Colzato LS, Prinz W, Hommel B (2010) The virtual co-actor: the social Simon effect does not rely on online feedback from the other. Front Psychol 1:208

Warneken F, Tomasello M (2006) Altruistic helping in human infants and young chimpanzees. Science 311:1301-1303

Welsh TN (2009) When $1+1=1$ : the unification of independent actors revealed through joint Simon effects in crossed and uncrossed effector conditions. Hum Mov Sci 28:726-737

Welsh TN, Lyons J, Weeks DJ, Anson JG, Chua R, Mendoza J, Elliott D (2007) Within- and between-nervous-system inhibition of return: observation is as good as performance. Psychon Bull Rev 14:950-956

Zajonc RB (1965) Social facilitation. Science 149:269-274 